\title{
Correlation between the expression of S100A4 and the efficacy of TAC neoadjuvant chemotherapy in breast cancer
}

\author{
WEN-LEI LI ${ }^{1,2 *}$, YANG ZHANG ${ }^{2 *}$, BAO-GUO LIU ${ }^{2}$, QIAN DU $^{3}$, CHANG-XIN ZHOU $^{2}$ and XING-SONG TIAN ${ }^{1}$ \\ ${ }^{1}$ Department of Breast and Thyroid Surgery, Shandong Provincial Hospital, Shandong University, \\ Jinan, Shandong 250021; Departments of ${ }^{2}$ Breast and Thyroid Surgery and ${ }^{3}$ Pediatrics, \\ People's Hospital of Liaocheng, Liaocheng, Shandong 252000, P.R. China
}

Received December 16, 2014; Accepted August 3, 2015

DOI: $10.3892 /$ etm.2015.2743

\begin{abstract}
The aim of this study was to investigate the correlation between the expression of S100A4 and the efficacy of neoadjuvant chemotherapy in breast cancer. A total of 65 patients with invasive breast cancer were treated with neoadjuvant chemotherapy using the TAC regimen. The expression of S100A4 was detected by an immunohistochemical two-step method prior to treatment, after 2 cycles of chemotherapy and after 4 cycles of chemotherapy. Pathological evaluations of the chemotherapy were performed using the Miller and Payne (MP) grading system and their correlation with the changes of S100A4 expression during and after the treatment were explored. Between pre-neoadjuvant chemotherapy and 4 cycles post-chemotherapy, there was a significant difference in the expression of S100A4 ( $<<0.05)$; S100A4 expression was associated with neoadjuvant chemotherapy. However, between pre-neoadjuvant chemotherapy and 2 cycles post-chemotherapy, there was no significant difference in the expression of S100A4 ( $\mathrm{P}>0.05)$. The intensity and changes of S100A4 expression were positively correlated with the efficacy of neoadjuvant chemotherapy $(\mathrm{r}=0.259, \mathrm{P}<0.05)$. When patients with an MP grade of I or II following the second cycle of neoadjuvant chemotherapy were continually treated with the original chemotherapy for another 2 cycles, the desired effect was generally not achieved. S100A4 may be used as a predictor of the efficacy of neoadjuvant chemotherapy in breast cancer, guiding the formulation of individualized programs to improve the effectiveness of the treatment. For patients with an MP grade level of I or II after 2 cycles of neoadjuvant chemo-
\end{abstract}

Correspondence to: Dr Xing-Song Tian, Department of Breast and Thyroid Surgery, Shandong Provincial Hospital, Shandong University, 324 Jingwuweiqi Road, Jinan, Shandong 250021, P.R. China

E-mail: xingsongtian@126.com

*Contributed equally

Key words: breast cancer, neoadjuvant chemotherapy, high frequency color Doppler ultrasound, coarse needle biopsy, S100A4 therapy, the use of alternative chemotherapy regimens should be considered.

\section{Introduction}

Neoadjuvant chemotherapy is an important part of the treatment of breast cancer. It not only greatly improves the effectiveness of surgical treatment and thereby increases the success rate of breast conservation, but also suppresses systemic subclinical metastases to a certain degree, and may improve the survival rate of patients (1). However, a variety of issues associated with neoadjuvant chemotherapy require further investigation, such as the identification of molecular biological markers to predict the efficacy of neoadjuvant chemotherapy. In particular, S100A4 has attracted a significant amount of attention from researchers (2).

S100A4 is a member of the S100 family of proteins; its expression is associated with the movement, invasion, metastasis, apoptosis and prognosis of various tumors $(3,4)$. Studies have demonstrated that pathophysiological progression is associated with S100A4 in breast cancer $(5,6)$, ovarian cancer (7), colon cancer (8), bladder cancer (9) and melanoma (10), which is closely associated with tumor incidence and metastasis. Currently, there are few studies concerning the correlation between the efficacy of neoadjuvant chemotherapy and the expression and changes of S100A4 (11-13), and the conclusions are inconsistent. In the present study, tumor tissues were collected after double coarse-needle biopsies; and the levels of S100A4 protein were detected, in order to explore the potential of S100A4 as molecular biological indicator for predicting the effect of neoadjuvant chemotherapy and guiding individual treatment.

\section{Materials and methods}

Patients and samples. A group of 65 female patients admitted to the department of breast and thyroid surgery of the People's Hospital of Liaocheng (Liaocheng, China) between October 2012 and December 2013 were included in the study. These patients, who were 22-63 years (mean, 42 years) old, were investigated using color ultrasound-guided coarse needle biopsy and diagnosed as having invasive breast cancer. Breast lumps were palpable in the clinical examination. The selected 
patients, who did not receive any pre-treatment, were treated with 4 cycles of TAC neoadjuvant chemotherapy prior to surgery. This study was approved by the ethics committee of the People's Hospital of Liaocheng. Informed consent was obtained from all patients.

Time and method of specimen collection. Hollow needle biopsy was performed respectively prior to neoadjuvant chemotherapy and following 2 cycles of neoadjuvant chemotherapy; surgical resection was performed following 4 cycles of neoadjuvant chemotherapy.

Neoadjuvant chemotherapy. A TAC regimen was administered, with each cycle comprising $75 \mathrm{mg} / \mathrm{m}^{2} \mathrm{~d} 1$ docetaxel, $50 \mathrm{mg} / \mathrm{m}^{2} \mathrm{~d} 1$ doxorubicin and $500 \mathrm{mg} / \mathrm{m}^{2} \mathrm{~d} 1$ cyclophosphamide for 21 days (d1 indicates that a single dose of the treatment was administered on day 1 of the treatment cycle).

Efficacy evaluation of neoadjuvant chemotherapy. The diameter and size of the tumor were measured with calipers and breast high frequency color Doppler ultrasound prior to neoadjuvant chemotherapy; the maximum diameters of the tumors of all patients were measured prior to and following neoadjuvant chemotherapy by the same operator with the same method of measurement; the results were recorded every 2 cycles.

Clinical efficacy was assessed using the RECIST evaluation criteria of Solid Tumors (14), and the results were divided into: Complete remission (CR), all target lesions disappeared and no new lesions appeared, tumor markers were normal, and were maintained for $\geq 4$ weeks; partial remission (PR), the maximum diameter sum of the target lesions decreased by $>30 \%$, and was maintained for $\geq 4$ weeks; stable disease (SD), the maximum diameter sum of the target lesions decreased less than that for PR, or increased less than that for disease progression (PD); $\mathrm{PD}$, the maximum diameter sum of the target lesions increased $\geq 20 \%$, and the absolute value increased by $\geq 5 \mathrm{~mm}$; the emergence of new lesions was also considered as PD. If only one of the longest diameters of the target lesions increased by $\geq 20 \%$, and the sum of the longest diameters of all recorded target lesions increased by $<20 \%$, this was not evaluated as PD. No residual cancer or only in situ carcinoma in samples after surgery was evaluated as pathological complete response (pCR). $\mathrm{CR}+\mathrm{PR}$ were considered effective; $\mathrm{SD}+\mathrm{PD}$ were considered invalid.

Pathologic evaluation of neoadjuvant chemotherapy was conducted using the Miller and Payne (MP) grading standards (15): Grade I, tumor lesions almost unchanged; grade II, a small number of tumor cells disappeared $(\leq 30 \%)$; grade III, most of the tumor tissues disappeared (30-90\%); grade IV, most tumors disappeared ( $>90 \%)$; grade $\mathrm{V}$, no residual invasive carcinoma. Grades III-V represented effective chemotherapy; grades I and II represented ineffective chemotherapy; and grade $\mathrm{V}$ represented a pathological complete response (pCR).

Immunohistochemistry. Dewaxed and hydrated tissue sections were placed in a pressure cooker containing citric tissue antigen retrieval solution for 3 min heat reparation; S100 A4 rabbit anti-human polyclonal antibody (1:200; ZA-0257; Beijing Zhongshan Golden Bridge Biotechnology Co., Ltd.,
Table I. Clinical evaluation after neoadjuvant chemotherapy.

\begin{tabular}{lcc}
\hline Efficacy & After 2 cycles, n $(\%)$ & After 4 cycles, n (\%) \\
\hline CR & $8(12.31)$ & $18(27.69)$ \\
PR & $39(60.00)$ & $31(47.69)$ \\
SD & $18(27.69)$ & $16(24.62)$ \\
PD & 0 & 0 \\
\hline
\end{tabular}

Results are for 65 patients with breast cancer. CR, complete remission; $\mathrm{PR}$, partial remission; $\mathrm{SD}$, stable disease; $\mathrm{PD}$, disease progression. For clinical evaluation of the efficacy of chemotherapy, CR and PR were effective and SD and PD were invalid.

Beijing, China) was added as the primary antibody, and then reagents 1 and 2 of the secondary antibody PV9000 detection system (ZSBIO, Beijing, China) were dropped in order. The tissues were then stained with DAB solution and sealed. Positive control samples underwent identical tonsil slice detection, tonsil lymphocytes appear in the cytoplasm as yellow or brown coloration, while stromal cells and vascular endothelial cells exhibited no yellow or brown coloring. Negative control samples were incubated with phosphate-buffered saline solution (blank reagent) instead of rabbit anti-human S100A4 antibody.

Evaluation of S100A4 expression. S100A4 positive expression was observed as brown cytoplasm, which was determined by a semi-quantitative scoring method in accordance with the cytoplasmic color intensity and number of positive cells; five visual fields of each slice were randomly selected under a microscope at a magnification of x200 (100 tumor cells per visual field) and the percentage of positive cells was recorded according to the degree of staining (A) and the proportion of stained cells (B) as follows: (A) degree of staining: no staining, 0 points; pale yellow, 1 point; brown, 2 points; and tan, 3 points. (B) proportion of stained cells: $<5 \%, 0$ points; 5 to $25 \%, 1$ point; $26-50 \%, 2$ points; $51-75 \%, 3$ points; $>75 \%, 4$ points. An integral $\mathrm{A}+\mathrm{B} \geq 2$ was considered to be $\mathrm{S} 100 \mathrm{~A} 4$ positive. Specifically: $0-1$ points was negative (-); 2 or 3 points was weakly positive (1+); 4 or 5 points represented moderately positive $(2+)$; and 6 or 7 points represented strongly positive (3+).

Statistical analysis. The data were statistically analyzed using the SPSS software package, version 17.0 (SPSS, Inc., Chicago, IL, USA). Changes of the expression of S100A4 before, during and after neoadjuvant chemotherapy were analyzed by Wilcoxon signed rank sum test; the correlation between the expression intensity of S100A4 and the effect of neoadjuvant chemotherapy was analyzed by $\chi^{2}$ and Spearman's rank correlation tests; $\mathrm{P}<0.05$ was considered to indicate a statistically significant result.

\section{Results}

Efficacy evaluation of neoadjuvant chemotherapy

Clinical evaluation of neoadjuvant chemotherapy. Following 2 cycles of neoadjuvant chemotherapy there were 47 cases of 
Table II. Pathological grading after neoadjuvant chemotherapy.

\begin{tabular}{lcc}
\hline Grade & After 2 cycles, $\mathrm{n}(\%)$ & After 4 cycles, $\mathrm{n}(\%)$ \\
\hline I & $9(13.85)$ & $7(10.77)$ \\
II & $13(20.00)$ & $12(18.46)$ \\
III & $37(56.92)$ & $19(29.23)$ \\
IV & $6(9.23)$ & $13(20.00)$ \\
V & 0 & $14(21.54)$ \\
\hline
\end{tabular}

Results are Miller and Payne grades for 65 patients with breast cancer. Grades III-V represented effective chemotherapy; grades I and II represented ineffective chemotherapy; grade V represented a pathological complete response.

Table III. Pathological grading of samples after 2 and 4 cycles of neoadjuvant chemotherapy.

\begin{tabular}{lcccc}
\hline & \multicolumn{2}{c}{ Pathological evaluation } & & \\
\cline { 2 - 3 } Time of sampling & Effective & Ineffective & $\chi^{2}$ & P-value \\
\hline After 2 cycles & 43 & 22 & 0.32 & $>0.05$ \\
After 4 cycles & 46 & 19 & & \\
\hline
\end{tabular}

Pathological evaluation based in Miller and Payne grades for 65 patients with breast cancer. Grades III-V represented effective chemotherapy and grades I and II represented ineffective chemotherapy.

effective chemotherapy, with an overall response rate of 47/65 (72.31\%); 8 cases had clinical CR (12.31\%). Following 4 cycles of neoadjuvant chemotherapy, there were 49 cases of effective chemotherapy, with an overall effective rate of 49/65 (75.38\%), 18 cases had clinical CR $(27.69 \%)$, and 16 patients $(24.62 \%)$ were not sensitive to neoadjuvant chemotherapy. Clinical evaluation results are shown in Table I and Fig. 1.

Pathologic evaluation of neoadjuvant chemotherapy. Following 2 cycles of neoadjuvant chemotherapy, MP pathological grading of the 65 patients was performed. There were 9 cases of grade I, 13 cases of grade II, 37 cases of grade III, 6 cases of grade IV and 0 cases of grade V; the chemotherapy response rate was $43 / 65(66.15 \%)$ and no pCR was observed. Following 4 cycles of neoadjuvant chemotherapy, there were 7 cases of grade I, 12 cases of grade II, 19 cases of grade III, 13 cases of grade IV and 14 cases of grade V; the chemotherapy response rate was $46 / 65$ (70.77\%), and the proportion of patients achieving pCR was 14/65 (21.54\%). There were 7 patients who continued to have no sensitivity to neoadjuvant chemotherapy.

The pathological evaluation results of the coarse needle specimens following 2 cycles of neoadjuvant chemotherapy and surgical specimens after 4 cycles of neoadjuvant chemotherapy were compared using the $\chi^{2}$ test, which showed no statistically significant difference $\left(\chi^{2}=0.32, \mathrm{P}>0.05\right)$, indicating that for patients that were determined to have grade I or II disease by pathologic evaluation after 2 cycles of neoadjuvant chemotherapy, alternative chemotherapy regimens should be considered. The grading and effectiveness data are shown in Tables II and III and Figs. 2 and 3.

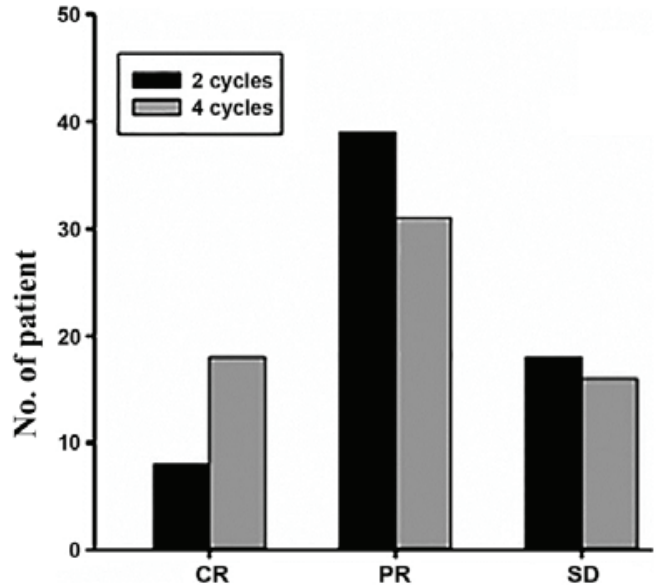

Figure 1. Clinical evaluation after neoadjuvant chemotherapy.

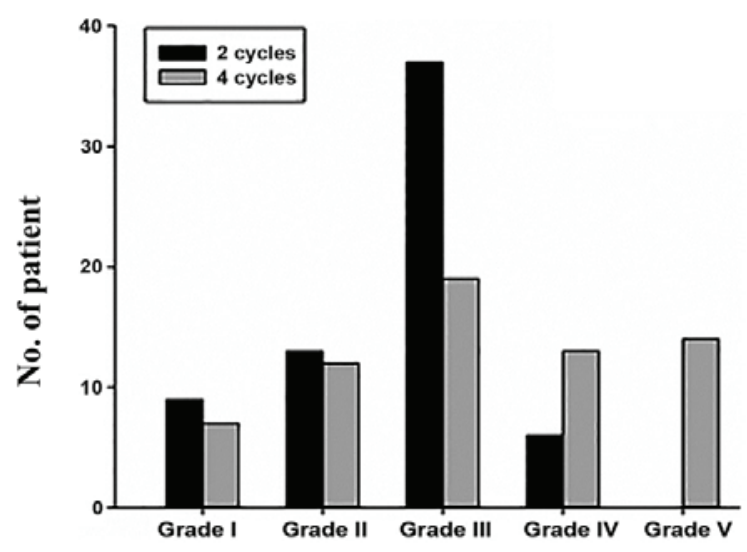

Figure 2. Miller and Payne pathological grading after neoadjuvant chemotherapy.

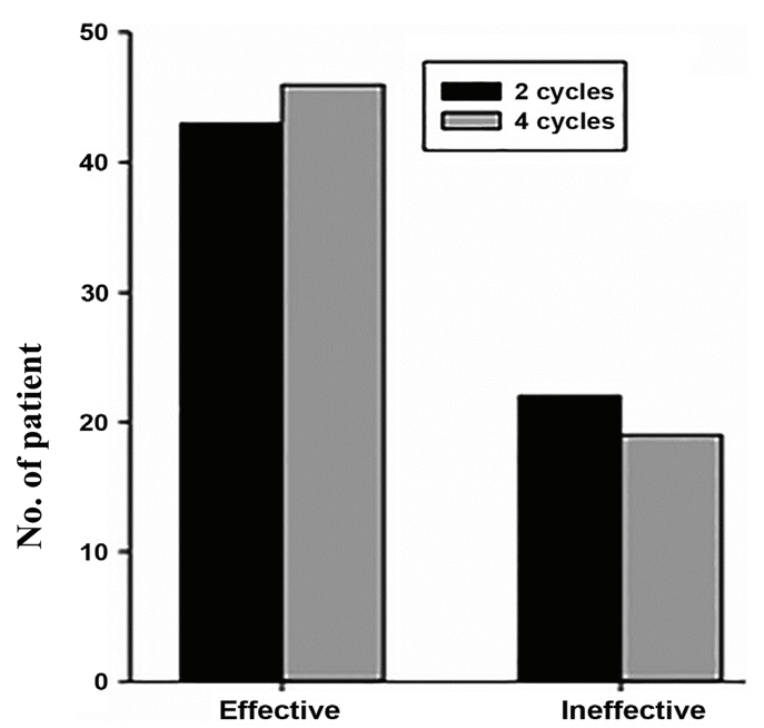

Figure 3. Miller and Payne pathological grading of samples after 2 cycles and 4 cycles of neoadjuvant chemotherapy.

S100A4 expression prior to neoadjuvant chemotherapy, and after 2 or 4 cycles of neoadjuvant chemotherapy. Among the 65 patients, prior to neoadjuvant chemotherapy, there 
Table IV. S100A4 expression prior to and after 4 cycles of breast cancer neoadjuvant chemotherapy.

\begin{tabular}{lrrrrr} 
& \multicolumn{4}{c}{ S100A4 expression after chemotherapy } \\
\cline { 2 - 5 } $\begin{array}{l}\text { S100A4 expression } \\
\text { prior to chemotherapy }\end{array}$ & - & + & ++ & +++ & P-value \\
\hline- & 11 & 5 & 2 & 1 & 0.032 \\
+ & 3 & 6 & 4 & 1 & \\
++ & 6 & 2 & 1 & 2 & \\
+++ & 3 & 2 & 1 & 1 \\
\hline
\end{tabular}

Table V. Change in 100A4 expression after 4 cycles of breast cancer neoadjuvant chemotherapy.

\begin{tabular}{lc}
\hline Change in S100A4 expression & $\mathrm{n}(\%)$ \\
\hline Increased & $15(29.41)$ \\
Unchanged & $19(37.26)$ \\
Decreased & $17(33.33)$ \\
\hline
\end{tabular}

were 20 patients who tested negative for S100A4 expression (30.77\%), 16 patients with weakly positive S100A4 expression (24.62\%), 15 patients with moderately positive S100A4 expression $(23.08 \%)$ and 14 patients with strongly positive S100A4 expression $(21.53 \%)$. Following 2 cycles of neoadjuvant chemotherapy, there were 22 cases negative for expression of S100A4 (33.85\%), 17 cases with weakly positive expression of S100A4 (26.15\%), 15 cases with moderately positive expression of S100A4 (23.08\%) and 11 cases with strongly positive expression of S100A4 (16.92\%). After 4 cycles of neoadjuvant chemotherapy, there were 23 cases negative for expression of S100A4 (45.10\%), 15 cases with weakly positive expression of S100A4 (29.41\%), 8 cases with moderately positive expression of S100A4 (15.69\%) and 5 cases with strongly positive expression of S100A4 (9.80\%). The remaining 14 patients achieved pathological complete remission after 4 cycles of neoadjuvant chemotherapy, and so it was not possible to determine S100A4 expression postoperatively. A comparison of 100A4 expression prior to chemotherapy and after 4 cycles of chemotherapy showed that expression was unchanged in 19 cases $(37.26 \%)$, increased in 15 cases $(29.41 \%)$ and decreased in 17 cases $(33.33 \%)$. Wilcoxon signed rank sum test showed that: There were significant differences in S100A4 expression between the time-points prior to neoadjuvant chemotherapy and following 4 cycles of neoadjuvant chemotherapy $(\mathrm{P}=0.032)$; there was no significant difference between pre-neoadjuvant chemotherapy and 2 cycles post-neoadjuvant chemotherapy $(\mathrm{P}>0.05)$; and neoadjuvant chemotherapy may lead to a reduction in the expression of S100A4 (Tables IV-VI).

Correlation between S100A4 expression and the efficacy of neoadjuvant chemotherapy. All 65 patients with breast cancer received neoadjuvant chemotherapy, and after 4 cycles of TAC neoadjuvant chemotherapy, no disease progression was observed. Routine MP grading evaluations and the expression levels of S100A4 protein for each grade as follows: Grade I,
7 cases (10.77\%); S100A4 expression was negative in 3 cases $(42.86 \%)$; weakly positive in 2 cases $(28.57 \%)$; moderately positive in 1 case $(14.29 \%)$; and strongly positive in 1 case (14.29\%); Grade II, 12 cases (18.46\%); S100A4 expression was negative in 8 cases $(66.67 \%)$; weakly positive in 3 cases (25.00\%); and moderately positive in 1 case $(8.33 \%)$; Grade III, 19 patients $(29.23 \%)$; S100A4 expression was negative in 6 cases $(31.58 \%)$; weakly positive in 7 cases $(36.84 \%)$; moderately positive in 3 cases $(15.79 \%)$; and strongly positive in 3 cases (15.79\%); Grade IV, 13 cases (20.00\%); S100A4 expression was negative in 2 cases $(15.38 \%)$; weakly positive in 2 cases (15.38\%); moderately positive in 6 cases $(46.15 \%)$, and strongly positive in 3 cases (23.09\%); Grade V, 14 (21.54\%); S100A4 expression was negative in 1 case $(7.14 \%)$; weakly positive in 2 cases (14.28\%); moderately positive in 4 cases (28.57\%); and strongly positive in 7 cases $(50.00 \%)$.

The results were analyzed by $\chi^{2}$ test and Spearman's rank correlation test, which showed that the efficacy of neoadjuvant chemotherapy was positively correlated with S100A4 expression $\left(\chi^{2}=7.46, \mathrm{P}<0.01\right)$; the higher the S100A4 expression, the better the efficacy of neoadjuvant chemotherapy $(r=0.259$, $\mathrm{P}<0.05)$, as shown in Tables VII and VIII.

\section{Discussion}

Since 1970 s, it has become widely accepted that breast disease is a systemic disease that can be hematogenous in the early stages. Therefore, treatment of breast cancer by surgery has gradually developed into a comprehensive treatment of the whole body. Chemotherapy and endocrine therapy, in particular, have greatly improved survival and quality of life and become the main methods used for the treatment of breast cancer, particularly for invasive breast cancer. Currently, neoadjuvant chemotherapy is recognized as the standard treatment for locally advanced breast cancer and inflammatory breast cancer by the medical profession. Its advantage is that it reduces local breast tumor size and controlling cancer invasion, thereby reducing tumor grade (16). It also provides data to support in vivo sensitivity testing in individuals to determine the effectiveness of a chemotherapy treatment plan. pCR, overall survival (OS) and disease-free survival (DFS) have improved significantly. Since the beginning of the 1980s, numerous neoadjuvant clinical studies of chemotherapy have been conducted. The National Surgical Adjuvant Breast and Bowel Project (NSABP) B-18 and B-27 protocols (1) and other experimental studies, revealed that postoperative chemotherapy and neoadjuvant chemotherapy in patients with breast 
Table VI. S100A4 expression prior to and after 2 cycles of breast cancer neoadjuvant chemotherapy.

\begin{tabular}{lrrrrr} 
& \multicolumn{4}{c}{ S100A4 expression after chemotherapy } \\
\cline { 2 - 5 } $\begin{array}{l}\text { S100A4 expression } \\
\text { prior to chemotherapy }\end{array}$ & - & + & ++ & +++ & 0 \\
\hline- & 14 & 5 & 1 & 0 & 0.214 \\
+ & 4 & 8 & 4 & 1 & \\
++ & 4 & 2 & 8 & 10 \\
+++ & 0 & 2 & 2 & 0 \\
\hline
\end{tabular}

Table VII. Correlation between S100A4 expression prior to chemotherapy and the efficacy of neoadjuvant chemotherapy in breast cancer.

\begin{tabular}{lllllll}
\hline & & \multicolumn{3}{c}{ S100A4 expression before chemotherapy } & \\
\cline { 3 - 5 } Chemotherapy efficacy & $\mathrm{n}$ & - & + & ++ & +++ & P-value \\
\hline Grade I & 7 & 3 & 2 & 1 & 1 & $<0.05$ \\
Grade II & 12 & 8 & 3 & 1 & 0 & \\
Grade III & 19 & 6 & 7 & 3 & 3 & \\
Grade IV & 13 & 2 & 2 & 6 & 3 & \\
Grade V & 14 & 1 & 2 & 4 & 7 & \\
\hline
\end{tabular}

Miller and Payne grades III-V represented effective chemotherapy and grades I and II represented ineffective chemotherapy.

Table VIII. Correlation between S100A4 expression prior to chemotherapy and the efficacy of neoadjuvant chemotherapy in breast cancer.

$$
\text { Efficacy, } n
$$

\begin{tabular}{lccc}
\cline { 2 - 3 } S100A4 expression & Effective & Ineffective & P-value \\
\hline Low & 20 & 26 & $<0.01$ \\
High & 16 & 3 &
\end{tabular}

Miller and Payne grades III-V represented effective chemotherapy and grades I and II represented ineffective chemotherapy. S100A4 staining: - and + represented low expression; ++ and +++ represented high expression.

cancer had no significant difference in OS and DFS during long-term follow-up (median follow-up, 16 years). However, if the patients reached pCR following neoadjuvant chemotherapy, both OS and DFS improved significantly.

At present, the evaluation of neoadjuvant chemotherapy is reliant upon clinical examination and pathological testing. The clinical evaluation includes specialist examination, breast ultrasound, mammography and magnetic resonance imaging (MRI). Among the currently advocated types of imaging examination, breast MRI is the primary option. Pathological evaluation, which involves the observation of postoperative specimens under a microscope to detect the apoptosis, degeneration and disappearance of tumor cells, is a more intuitive method with high reliability. Studies have shown that survival is significantly improved in patients with a pCR prognosis $(1,17)$. Therefore, pathological assessment is an important method for the evaluation of neoadjuvant chemotherapy. However, both neoadjuvant chemotherapy clinical assessment and pathological assessment have defects. The identification of predictors of the efficacy of neoadjuvant chemotherapy is important for reducing the suffering and economic burden of chemotherapy, enabling other effective treatment methods to be sought, and improving patient survival and quality of life.

Since the advent of neoadjuvant chemotherapy, considerable research has been conducted to predict its efficacy. This is important for preventing chemotherapy-insensitive patients from undergoing neoadjuvant chemotherapy-induced progression. Early studies of neoadjuvant chemotherapy predictors mainly concerned clinical indicators. For example, the NSABP B-18 study (18) found that the histological grading of breast cancer was predictive for the efficacy of neoadjuvant chemotherapy. With the development of molecular biology, increasing attention has been focused on biological factors in breast cancer for predicting the efficacy of neoadjuvant chemotherapy. However, at present, that has been no consensus on any of the predictors that have been proposed. However, they have laid the foundation for further studies on predictive methods and predictors of neoadjuvant chemotherapy efficacy.

S100A4, also known as Mts1, pEL298, 18A2, 42A, p9Ka, calvasculin, CAPL and FSP1, has a molecular weight of $11.5 \times 10^{3}$, and is located in the long arm of chromosome zone 2 band 1 (1q21) (19). This zone is unstable; therefore chromosomal absence, translocation, duplication and other 
changes can easily occur, which are closely associated with the incidence, development and invasion of tumors. The S100A4 gene encodes a calcium-binding protein with a double EF helix, and is a member of the S100 calcium-binding protein superfamily. It is a metastasis-associated protein. A previous study found that S100A4 was capable of regulating the cell cycle and promoting invasion and metastasis (20). In addition, it is also associated with calcium signaling pathways for the regulation of the expression of genes associated with cell motility, adhesion, proliferation, differentiation, apoptosis and other pathophysiological processes. Albertazzi et al (21) found that in breast cancer, the expression level of S100A4 protein correlated with metastasis. Although studies have not shown directly that S100A4 plays an active role, liprin B1, methionine amino peptidase, P53 and certain proteins involved in cytoskeletal rearrangement and cell motility have been found to interact with the S100A4 protein, increasing the ability of tumor cells to become invasive and metastatic (3). Wang et al (22) detected the S100A4 protein by immunohistochemistry in colorectal cancer, adjacent normal mucosa, lymph node metastasis, liver cancer and colorectal adenomas. They found that its expression level in colorectal cancer was significantly higher than that in adjacent normal mucosa and adenoma $(\mathrm{P}<0.05)$. They also found that its expression levels in patients with lymph node metastasis and liver metastasis and at relatively-advanced Duke stage were significantly higher than those in patients without lymph node metastasis and liver metastasis and at an early Duke stage in colorectal cancer $(\mathrm{P}<0.05)$. Through study of the S100 family, Jin et al (23) found that the expression level of S100A4 protein was only associated with chemotherapy during chemotherapy $(\mathrm{P}<0.05)$. Ambartsumian et al (24) observed that S100A4 played an important role in promoting angiogenesis directly; it was involved in cancer development and metastasis throughout most of the pathophysiological process. Through the investigation of clinically obtained tissue samples, Rudland et al found that for breast cancer, the number of lymph node metastases and the scope and degree of malignancy were closely correlated with the expression level of S100A4 (6). By investigating the expression levels of S100A4 in Luminal A type breast cancer, Luminal B type breast cancer, breast cancer with HER-2 overexpression, basal-like breast cancer and adjacent breast carcinoma, Wang et al (25) found that the positive rate of S100A4 expression in tissues adjacent to breast cancer was $(45.0 \% ; 18 / 40)$, significantly lower than that in normal tissues $(62.0 \%$; 67/108) $(\mathrm{P}<0.05)$. Furthermore, in different molecular subtypes of breast tissue, the positive rate of S100A4 expression in HER-2-overexpressing and basal-like breast cancer tissues were higher than that in the Luminal A and Luminal B types $(\mathrm{P}<0.05)$. S100A4 exhibited high expression levels in breast cancer patients with lymph node metastasis $(\mathrm{P}<0.05)$.

Clinical studies $(26,27)$ have shown that changes in the expression of certain genes could predict the efficacy of neoadjuvant chemotherapy. By comparing the expression of the estrogen receptor (ER), progesterone receptor (PR) and HER-2 in 43 patients receiving TAC neoadjuvant chemotherapy before chemotherapy and after surgery, Li et al (28) found that the expression levels of ER, PR and HER-2 did not significantly change during the course of chemotherapy $(P>0.05)$, but observed that ER and/or PR-negative breast cancer patients were more sensitive to chemotherapy. MacGrogan et al (29) found that in patients with no or low ER expression and high Ki67 expression, the efficacy of chemotherapy was likely to be improved. Zhou et al (30) reported that neoadjuvant chemotherapy is able to reduce the expression of $\mathrm{Ki} 67$, but has insignificant effects on the expression of ER, PR and HER-2. Zhao et al (31) conducted a retrospective analysis of the correlation between the expression of ER, PR, p53 and Bcl-2 and the efficacy of neoadjuvant chemotherapy in 98 cases of breast cancer, and found that the expression of p53 and Bcl-2 changed significantly following chemotherapy $(\mathrm{P}<0.05)$; as the effect of chemotherapy was increased, the expression of p53 decreased, and the expression intensity of $\mathrm{Bcl}-2$ was positively correlated with chemotherapy efficacy $(\mathrm{P}<0.05)$. However, the expression levels of ER and PR did not significantly change from their pretreatment levels after chemotherapy. According to these previous studies, neoadjuvant chemotherapy can reduce the expression of $\mathrm{Ki} 67$ and $\mathrm{p} 53$, and enhance the expression of Bcl-2, but has no significant effect on the expression of ER, PR and HER-2,

The present study analyzed the correlation between the expression of S100A4 protein prior to neoadjuvant chemotherapy and the efficacy of neoadjuvant chemotherapy. It was observed that in the 65 patients receiving neoadjuvant chemotherapy, there were 46 cases $(70.77 \%)$ in which chemotherapy was effective, of which 36 cases expressed S100A4 protein (78.26\%) and 10 cases did not $(21.74 \%)$. Chemotherapy was ineffective in 19 cases, 8 of whom tested positive for S100A4 protein expression $(42.10 \%)$ and 11 of whom tested negative $(57.90 \%)$. There were significant differences in the efficacy of neoadjuvant chemotherapy between positive and negative S100A4 expression groups $(\mathrm{P}<0.05)$. Furthermore, the efficacy of neoadjuvant chemotherapy was positively correlated with $\mathrm{S} 100 \mathrm{~A} 4$ protein expression $(\mathrm{r}=0.259, \mathrm{P}<0.05)$; the higher the S100A protein expression, the better the efficacy of neoadjuvant chemotherapy. In the present study, in the 14 patients with a pCR, there were 9 cases $(64.29 \%)$ with strongly positive expression of S100A4 protein (+++) and 2 cases (14.29\%) that were negative for S100A4 protein expression, a significantly smaller proportion than those who were positive for S100A4 protein expression. The reasons for this are hypothesized to be as follows: S100A4 protein expression is closely associated with the degree of tumor differentiation and malignancy; with a high degree of differentiation, low-grade malignant tumor cells are not sensitive to chemotherapy and have low S100A4 expression. By contrast, less-differentiated and highly malignant tumor cells highly express S100A4 protein and are also more sensitive to chemotherapy.

In this study, S100A4 expression in 65 patients with breast cancer was detected prior to, during (following 2 cycles) and after (following 4 cycles) of neoadjuvant chemotherapy; it was observed that there were significant differences in S100A4 expression prior to, during and after neoadjuvant chemotherapy, particularly between the expression levels prior to and following neoadjuvant chemotherapy (all $\mathrm{P}<0.05$ ); and a reduction in $\mathrm{S} 100 \mathrm{~A} 4$ expression was associated with an enhanced chemotherapeutic effect. Although the mechanism remains incompletely elucidated, it may involve the following: i) S100A4 protein is secreted by tumor cells and tumor-activated stromal cells; when neoadjuvant chemo- 
therapy was administered, tumor cells and tumor-activated stromal cells were continually killed, leading to the decreased expression of S100A4; ii) neoadjuvant chemotherapy destroys the original structure of DNA so that genetic mutations or gene rearrangements occur, resulting in the damage of the S100A4 gene, thereby reducing the expression of S100A4 protein. However, further studies are required to fully explore the underlying mechanism.

\section{References}

1. Rastogi P, Anderson SJ, Bear HD, Geyer CE, Kahlenberg MS, Robidoux A, Margolese RG, Hoehn JL, Vogel VG, Dakhil SR, et al: Preoperative chemotherapy: Updates of national surgical adjuvant breast and bowel project protocols B-18 and B-27. J Clin Oncol 26: 778-785, 2008.

2. Schmidt-Hansen B, Klingelhöfer J, Grum-Schwensen B, Christensen A, Andresen S, Kruse C, Hansen T, Ambartsumian N, Lukanidin E and Grigorian M: Functional significance of metastasis-inducing S100A4(Mts1) in tumor-stroma interplay. J Biol Chem279: 24498-24504, 2004

3. Garrett SC, Varney KM, Weber DJ and Bresnick AR: S100A4, a mediator of metastasis. J Biol Chem 281: 677-680, 2006.

4. Emberley ED, Murphy LC and Watson PH: S100 proteins and their influence on pro-survival pathways in cancer. Biochem Cell Biol 82: 508- 515, 2004

5. Lee WY, Su WC, Lin PW, Guo HR, Chang TW and Chen HH: Expression of S100A4 and Met: Potential predictors for metastasis and survival in early-stage breast cancer. Oncology 66: 429-438, 2004

6. Rudland PS, Platt-Higgins A, Renshaw C, West CR Winstanley JH, Robertson L and Barraclough R: Prognostic significance of the metastasis-inducing protein S100A4 (p9Ka) in human breast cancer. Cancer Res 60: 1595-1603, 2000.

7. Kikuchi N, Horiuchi A, Osada R, Imai T, Wang C, Chen X and Konishi I: Nuclear expression of S100A4 is associated with aggressive behavior of epithelial ovarian carcinoma: An important autocrine/paracrine factor in tumor progression. Cancer Sci 97: 1061-1069, 2006.

8. Kim JH, Kim CN, Kim SY, Lee JS, Cho D, Kim JW and Yoon SY: Enhanced S100A4 protein expression is clinic pathologically significant to metastatic potential and p53 dysfunction in colorectal cancer. Oncol Rep 22: 41-47, 2009.

9. Agerbaek M, Alsner J, Marcussen N, Lundbeck F and Von der Maase H: Focal S100A4 protein expression is an independent predictor of development of metastatic disease in cystectomized bladder cancer patients. Eur Urol 50: 777-785, 2006.

10. Andersen K, Nesland JM, Holm R, Flørenes VA, Fodstad Ø and Maelandsmo GM: Expression of S100A4 combined with reduced E-cadherin expression predicts patient outcome in malignant melanoma. Mod Pathol 17: 990-997, 2004.

11. Taylor S, Herrington S, Prime W, Rudland PS and Barraclough R: S100A4(p9Ka) protein in colon carcinoma and liver metastases: Association with carcinoma cells and T-lymphocytes. $\mathrm{Br}$ J Cancer 86: 409-416, 2002.

12. Komatsu K, Murata K, Kameyama M, Ayaki M, Mukai M, Ishiguro S, Miyoshi J, Tatsuta M, Inoue M and Nakamura $H$ : Expression of S100A6 and S100A4 in matched samples of human colorectal mucosa, primary colorectal adenocarcinomas and liver metastases. Oncology 63: 192-200, 2002.

13. Kriajevska M, Tarabykina S, Bronstein I, Maitland N, Lomonosov M, Hansen K, Georgiev G and Lukanidin E: Metastasis-associated Mts1(S100A4) protein modulates protein kinase $\mathrm{C}$ phosphorylation of the heavy chain of nonmuscle myosin. J Biol Chem 273: 9852-9856, 1998.

14. Chen ZW and Liao ML: RECIST criteria used in evaluating the efficacy of cancer therapy. Zhong Guo Ai Zheng 10: 6-8, 2004 (In Chinese).
15. Corben AD, Abi-Raad R, Popa I, Teo CH, Macklin EA, Koerner FC, Taghian AG and Brachtel EF: Pathologic response and long-term follow-up in breast cancer patients treated with neoadjuvant chemotherapy: A comparison between classifications and their practical application. Arch Pathol Lab Med 137: 1074-1082, 2013.

16. Liu G and Wang YS: Progress of breast-conserving surgery after neoadjuvant chemotherapy for breast cancer. Zhongguo Pu Wai Ji Chu Yu Lin Chuang Za Zhi 17: 1249-1252, 2010 (In Chinese).

17. Ring AE, Smith IE, Ashley S, Fulford LG and Lakhani SR: Oestrogen receptor status, pathological complete response and prognosis in patients receiving neoadjuvant chemotherapy for early breast cancer. Br J Cancer 91: 2012-2017, 2004.

18. Fisher ER, Wang J, Bryant J, Fisher B, Mamounas E and Wolmark N: Pathbiology of preoperative chemotherapy: Findings from the national surgical adjuvant breast and bowel (NSABP) protocol B-18. Cancer 95: 681-695, 2002.

19. Zhang J, Zhang D L, Jiao XL and Dong Q: S100A4 regulates migration and invasion in hepatocellular carcinoma HepG2 cells via NF- $\mathrm{BB}-$ dependent MMP-9 signal. Eur Rev Med Pharmacol Sci 17: 2372-2382, 2013

20. Helfman DM, Kim EJ, Lukanidin E and Grigorian M: The metastasis associated protein S100A4: Role in tumor progression and metastasis. Br J Cancer 92: 1955-1958, 2005.

21. Albertazzi E, Cajone F, Leone BE, Naguib RN, Lakshmi MS and Sherbet GV: Expression of metastasis-associated genes h-mts1 (S100A4) and nm23 in carcinoma of breast is related to disease progression. DNA Cell Biol 17: 335-342, 1998.

22. Wang XS, Lin SS, Wang GY, Zou DL, Chen HS and You Q: Correlation between S100A4 and E-Cad protein expression and invasion, metastasis and prognosis of colorectal cancer. Zhongguo Zhong Liu Lin Chuang 36: 690-693, 2009 (In Chinese).

23. Jin L, Shen Q, Ding S, Jiang W, Jiang L and Zhu X: Immunohistochemical expression of Annexin A2 and S100A proteins in patients with bulky stage IB-IIA cervical cancer treated with neoadjuvant chemotherapy. Gynecol Oncol 126: 140-146, 2012.

24. Ambartsumian N, Klingelhöfer J, Grigorian M, Christensen C, Kriajevska M, Tulchinsky E, Georgiev G, Berezin V, Bock E, Rygaard J, et al: The metastasis-associated Mts1 (S100A4) protein could act as an angiogenic factor. Oncogene 20: 4685-4695, 2001.

25. Wang Q, Yu MH, Wang G and Wang MH: Expression and clinical significance of DLL4 and S100A4 in different molecular subtypes of breast carcinoma. Zhongguo Pu Wai Ji Chu Yu Lin Chuang Za Zhi 19: 957-961, 2012 (In Chinese)

26. Chang JC, Wooten EC, Tsimelzon A, Hilsenbeck SG, Gutierrez MC, Elledge R, Mohsin S, Osborne CK, Chamness GC, Allred DC and O'Connell P: Gene expression profiling for the prediction of therapeutic response to docetaxel in patients with breast cancer. Lancet 362: 362-369, 2003.

27. Hess KR, Anderson K, Symmans WF, Valero V, Ibrahim N, Mejia JA, Booser D, Theriault RL, Buzdar AU, Dempsey PJ, et al: Pharmacogenomic predictor of sensitivity to preoperative chemotherapy with paclitaxel and fluorouracil, doxorubicin and cyclpophosphamide in breast cancer. J Clin Oncol 24: 4236-4244, 2006.

28. Li SJ, Han B, Fu T, Shi AP, Wu D, Liu GJ and Fan ZM: The relationship between ER, PR and the expression of HER-2 and breast cancer neoadjuvant chemotherapy reaction. Zhong Guo Lao Nian Xue Za Zhi 29: 474-476, 2009 (In Chinese).

29. MacGrogan G, Mauriac L, Durand M, Bonichon F, Trojani M, de Mascarel I and Coindre JM: Primary chemotherapy in breast invasive carcinoma: Predictive value of the immunohistochemical detection of hormonal receptors, $\mathrm{p} 53$, c-erbB-2, MiB1, pS2 and GST pi. Br J Cancer 74: 1458-1465, 1996.

30. Zhou QH, Wu Y, Cai ZR and Zhu JX: Influence of neoadjuvant chemotherapy on ER, PR, C-erbB-2, Ki-67 expressions of breast cancer patients. Zhong Guo Ai Zheng Za Zhi 18: 139-141, 2008 (In Chinese).

31. Zhao YC, Li Y, Zhu YY and Luo CY: Significance of expression changes of ER, PR, p53 and Bcl-2 induced by neoadjuvant chemotherapy (NAC) in breast cancer. Xian Dai Zhong Liu Yi Xue 19: 2017-2020,2011 (In Chinese). 